\title{
Lasing threshold and mode competition in chaotic cavities
}

\author{
T. Sh. Misirpashaev ${ }^{1,2}$ and C. W. J. Beenakker ${ }^{1}$ \\ ${ }^{1}$ Instituut-Lorentz, University of Leiden, P.O. Box 9506, 2300 RA Leiden, The Netherlands \\ ${ }^{2}$ Landau Institute for Theoretical Physics, 2 Kosygin Street, Moscow 117334, Russia
}

(Received 4 April 1997; revised manuscript received 22 August 1997)

\begin{abstract}
The lasing threshold of a multimode chaotic cavity (linear size $D \gg$ wavelength $\lambda$ ) coupled to the outside through a small hole (linear size $d \ll \lambda$ ) is studied. For sufficiently weak absorption by the boundaries, the statistical distribution of the threshold is wide, its mean value being much less than the pumping rate needed to compensate the average loss. The average number $\left\langle N_{\mathrm{nc}}\right\rangle \gg 1$ of noncompeting excited modes is proportional to the square root of the pumping rate. We use the classical model of spatial hole burning to account for mode competition and find a reduction in the average number of excited modes to $\langle N\rangle=3^{1 / 3}\left\langle N_{\mathrm{nc}}\right\rangle^{2 / 3}$. [S1050-2947(98)06802-4]

PACS number(s): 42.55.Sa, 05.45. $+\mathrm{b}$, 42.55.Ah, 78.45. $+\mathrm{h}$
\end{abstract}

\section{INTRODUCTION}

Incorporation of quantum optical effects is a necessary and interesting extension of active ongoing research on multiple scattering of electromagnetic waves in random media [1]. It becomes particularly important when the medium is active, as is the case in experimentally realized "random lasers" [2-4]. Quantum effects have been largely ignored in many publications devoted to propagation in disordered amplifying waveguides [5-8], in which only amplified stimulated emission of external incoming flux but not of spontaneously emitted internal noise was taken into account (Ref. [9] being a notable exception). Amplified internal noise can lead to excitation of low-threshold lasing modes of the waveguide, making practical use of amplifying waveguides problematic. The difficulty of the waveguide geometry is the onset of localization. In this paper we consider a simpler cavity geometry, which does not show localization, but retains two essential features of the problem: (1) large sample-to-sample fluctuations and (2) instability brought about by spontaneous emission.

A complete description of the fluctuations is possible in the universal regime characterized by a chaotic pattern of classical trajectories. We assume that the cavity (volume $V$ $\simeq D^{3}$ ) is confined by conducting walls, filled with a lasing medium (central frequency of the gain profile $\omega_{0}$ ), and coupled to external detectors via one or several small holes. It was demonstrated recently that a nonintegrable shape of the resonator can significantly affect its lasing properties [10]. Chaoticity of classical trajectories can be achieved either by a peculiar shape of the resonator [11-13], or by a small amount of disorder scattering. We will speak about "chaotic cavities,', meaning either of the two mechanisms responsible for the onset of chaos.

We restrict ourselves to the case of well-resolved cavity modes, which means that (1) resistive loss $\gamma_{*}$ in the cavity walls is less than the mean modal spacing $\delta \omega_{0}=\pi^{2} c^{3} / \omega_{0}^{2} V$ and (2) characteristic size of the holes $d$ is smaller than the wavelength $\lambda_{0}=2 \pi c / \omega_{0}$. Mean loss $\gamma_{0}$ through a small hole was calculated by Bethe [14],

$$
\gamma_{0} \simeq \frac{c d^{6}}{\lambda_{0}^{4} V}, \quad d \ll \lambda_{0},
$$

so that $\gamma_{0} / \delta \omega_{0} \simeq\left(d / \lambda_{0}\right)^{6} \ll 1$. Note that the loss (1) is not proportional to the area of the hole. It is in fact much smaller than one might guess by extrapolating the dependence $\gamma_{0}$ $\simeq c d^{2} / V$ valid for $d \gg \lambda_{0}$. The effect of sample-to-sample fluctuations is pronounced only if $\gamma_{*} \ll \gamma_{0}$. This regime is experimentally accessible, as was demonstrated by a recent series of experiments on microwave cavities with superconducting niobium walls $[12,13]$.

Each act of spontaneous emission in a pumped cavity is a source of radiation into some cavity mode. Classical condition of the lasing threshold in a given cavity mode is satisfied if the gain due to stimulated emission equals the loss. Threshold for the cavity is the smallest value of the pumping rate at which threshold is attained for one of the modes. The questions we ask are, what is the threshold rate of pumping? How many lasing modes can coexist for a given pumping rate above the threshold? The problem of spectral content of outgoing radiation has been widely studied for integrable cavities of definite shape. Considering arrays of chaotic cavities of slightly varying shape or with different configurations of scatterers we address the problem statistically and compute the probability of lasing, the distribution of the threshold, and the average number of excited modes.

Trivially, gain greater than mean loss $\gamma_{0}$ will be on the average sufficient to ensure lasing, while gain smaller than $\gamma_{*}$ will never suffice. The mean loss from a tiny hole is small. We argue that the actual average threshold can still be many orders of magnitude smaller. Each individual cavity exhibits a well-defined threshold but its statistical distribution is wide. In Sec. II we compute this distribution for the idealized case $\gamma_{*}=0$. Effects of nonzero resistivity of the walls are discussed in Sec. III. Section IV is devoted to the computation of the average number of excited modes above the threshold. We conclude in Sec. V.

\section{DISTRIBUTION OF LASING THRESHOLD}

We assume that the line of spontaneous emission is homogeneously broadened and has Lorentzian shape with central frequency $\omega_{0}$ and width $2 \Omega$. Let $\psi_{i}(\vec{r})$ be the amplitude of a mode of the closed cavity at frequency $\omega_{i}$, normalized according to $\int d \vec{r} \psi_{i}^{2}(\vec{r})=V$. (For simplicity we neglect polar- 
ization dependent phenomena and work with real scalar field amplitudes.) In the presence of weak coupling to the outside world the modes acquire finite widths $\gamma_{i}$. We assume two sets of conditions:

$$
\gamma_{0} \ll \delta \omega_{0}<\Omega \ll \omega_{0}, \quad d \ll \lambda_{0} \ll D .
$$

An especially important role is played by the inequality $\gamma_{0}$ $\ll \delta \omega_{0}$, which is implied by $d \ll \lambda_{0}$. It ensures that the modes of the open cavity are well defined and do not differ significantly from those of the closed one. In this section we consider the idealized case in which there is no loss in the walls of the cavity $\left(\gamma_{*}=0\right)$.

In a chaotic cavity the modes $\psi_{i}(\vec{r})$ can be modeled as random superpositions of plane waves [15]. (Validity of this model has been checked experimentally in microwave cavities $[12,16]$.) This implies a Gaussian distribution for $\psi_{i}(\vec{r})$ at any point $\vec{r}$. The corresponding distribution for $\psi_{i}^{2}(\vec{r})$ is called the Porter-Thomas distribution [17]. Loss from a small hole located at $\vec{r}$ is proportional to $\left[\vec{\nabla}_{\vec{n}} \psi_{i}(\vec{r})\right]^{2}$ [with $\vec{\nabla}_{\vec{n}} \psi_{i}(\vec{r})$ the derivative in the direction normal to the surface of the hole] and has the same Porter-Thomas distribution, which was directly probed in the experiments of Ref. [12]. More generally, the distribution of normalized modal widths $y$ $=\gamma_{i} / \gamma_{0}$ in a cavity with $\nu$ holes is given by the $\chi^{2}$ distribution with $\nu$ degrees of freedom (normalized to 1 ),

$$
P_{\nu}(y)=\frac{(\nu / 2)^{\nu / 2}}{\Gamma(\nu / 2)} y^{-1+\nu / 2} \exp (-\nu y / 2) .
$$

We assumed that loss from different holes is independent, which is true provided their separation is larger than $\lambda_{0}$. For small integer $\nu$, the distribution (3) is wide. The single-hole case $\nu=1$ looks especially promising from the point of view of low-threshold lasing because $P_{1}(y)=\exp (-y / 2) / \sqrt{2 \pi y}$ grows with decreasing $y$.

To grasp the picture we first confine ourselves to a subset of cavity modes located near $\omega_{0}$. We neglect fluctuations of their frequencies and assume that the modes are equidistant, $\omega_{m}=\omega_{0}+\delta \omega_{0} m, m=0, \pm 1, \pm 2, \ldots$. We denote by $R_{p_{0}}$ a reference pumping rate necessary to provide gain equal to the mean loss $\gamma_{0}$ at frequency $\omega_{0}$, and introduce the reduced pumping rate $\varepsilon=R_{p} / R_{p_{0}}$, assumed $\ll 1$. Loss of different modes is uncorrelated and distributed according to Eq. (3) while gain diminishes with increasing difference $\left|\omega-\omega_{0}\right|$ according to the Lorentzian

$$
g_{0}(\omega)=\gamma_{0} \varepsilon\left[1+\left(\omega-\omega_{0}\right)^{2} / \Omega^{2}\right]^{-1} .
$$

It follows that the probability $p_{\nu}(\varepsilon)$ of there being no lasing mode at the pumping rate $\varepsilon$ is given by

$$
p_{\nu}(\varepsilon)=\prod_{m}\left(1-\int_{0}^{g_{0}\left(\omega_{m}\right) / \gamma_{0}} d y P_{\nu}(y)\right) .
$$

For $\varepsilon \ll 1$, the upper limit of the integral is also $\ll 1$, and we can replace $P_{\nu}(y)$ by its leading behavior at small $y$, $P_{\nu}(y) \propto y^{-1+\nu / 2}$, which yields

$$
\begin{gathered}
p_{\nu}(\varepsilon) \approx \prod_{m}\left(1-\frac{C_{\nu} \varepsilon^{\nu / 2}}{\left[1+m^{2}\left(\delta \omega_{0} / \Omega\right)^{2}\right]^{\nu / 2}}\right) \\
\approx \exp \left(-C_{\nu} \varepsilon^{\nu / 2} \sum_{m}\left[1+m^{2}\left(\delta \omega_{0} / \Omega\right)^{2}\right]^{-\nu / 2}\right), \\
C_{\nu}=(\nu / 2)^{-1+\nu / 2}[\Gamma(\nu / 2)]^{-1} .
\end{gathered}
$$

Because the summand decays as $m^{-\nu}$ we find that for $\nu>1$ the leading behavior of the probability of no lasing is determined by the modes with $|m| \lesssim \Omega / \delta \omega_{0}$,

$$
p_{\nu}(\varepsilon) \approx \exp \left[-\widetilde{C}_{\nu}\left(\Omega / \delta \omega_{0}\right) \varepsilon^{\nu / 2}\right] .
$$

Here $\widetilde{C}_{\nu}=\sqrt{\pi} C_{\nu} \Gamma[(\nu-1) / 2] / \Gamma(\nu / 2)$ for $\nu>1$ (below we will separately define $\left.\widetilde{C}_{1}\right)$. Modes far from $\omega_{0}$ have negligible chance to get excited and need not be taken into account. On the contrary, for $\nu=1$ all cavity modes, including those very far from $\omega_{0}$, contribute to the probability.

To treat the contribution of distant modes for $\nu=1$ correctly, we must account for several factors which we could ignore for $\nu>1$. (1) The spectral density cannot be replaced by its value $\rho_{0}=1 / \delta \omega_{0}$ at $\omega=\omega_{0}$. Instead $\rho(\omega)=\rho_{0} \omega^{2} / \omega_{0}^{2}$. (2) The mean loss is frequency dependent, $\bar{\gamma}(\omega)=\gamma_{0} \omega^{4} / \omega_{0}^{4}$, cf. Eq. (1). (3) The Lorentzian (4) for the amplification rate is an approximation valid only in the vicinity of $\omega_{0}$. A correct expression for the gain $g(\omega)$ must be even in $\omega$ to comply with the symmetry $\chi(\omega)=\chi^{*}(-\omega)$ of the dielectric susceptibility $\chi$. It includes contributions of both poles $\pm \omega_{0}+i \Omega$ and reads

$$
g(\omega)=\frac{4 \omega^{2} \gamma_{0} \varepsilon \Omega^{2}}{\left(\omega^{2}-\omega_{0}^{2}\right)^{2}+2\left(\omega^{2}+\omega_{0}^{2}\right) \Omega^{2}+\Omega^{4}} .
$$

Taking these three factors into account and replacing the discrete sum by an integral, the probability of no lasing is given by

$$
p_{\nu}(\varepsilon)=\exp \left(\int_{0}^{\omega_{\max }} d \omega \rho(\omega) \ln \int_{g(\omega) / \bar{\gamma}(\omega)}^{\infty} d y P_{\nu}(y)\right) .
$$

For $\nu>1$ this leads to Eq. (8), the ultraviolet cutoff $\omega_{\max }$ being irrelevant. For $\nu=1$ we get

$$
p_{1}(\varepsilon)=\exp \left(\int_{0}^{\omega_{\max }} d \omega \rho(\omega) \ln \left[1-\operatorname{erf} \sqrt{\frac{g(\omega)}{2 \bar{\gamma}(\omega)}}\right]\right),
$$

where $\operatorname{erf}(z)=(2 / \sqrt{\pi}) \int_{0}^{z} d x \exp \left(-x^{2}\right)$ is the error function. The main logarithmic contribution of type $\int d \omega / \omega$ to the integral in Eq. (11) comes from large values of $\omega$. The ultraviolet cutoff $\omega_{\max } \simeq 2 \pi c / d$ appears because loss of high frequency modes with $\lambda<d$ no longer exhibits the strong fluctuations of Eq. (3). Beyond the cutoff classical ray optics applies, leading to a narrowly peaked distribution of the loss around the value $c d^{2} / V \gg \gamma_{0}$. Because we are considering the case $\varepsilon \ll 1$ in which the gain is much smaller than the average 


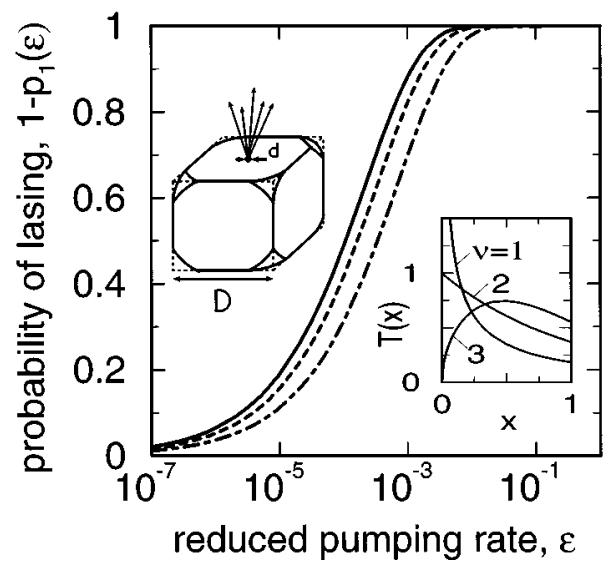

FIG. 1. Probability of lasing $1-p_{1}(\varepsilon)$ versus reduced pumping rate $\varepsilon$ with $p_{1}(\varepsilon)$ given by Eq. (11) $\left(\omega_{0} / \Omega=10, \Omega / \delta \omega_{0}=10\right)$. Thick lines are for different ratios $D / d$ corresponding to different numbers $M \simeq(D / d)^{3}$ of relevant cavity modes (dot-dashed line $M$ $=10^{2}$, dashed line $M=10^{3}$, solid line $M=10^{4}$ ). Left inset shows an example of chaotic cavity. Chaotic behavior of classical trajectories in this particular "die" shaped cavity was shown in Ref. [11]. Radiation is confined inside by means of ideally conducting walls and can leave the cavity only through a tiny hole. Right inset shows the probability distribution of the lasing threshold $T_{\nu}(x)=(\nu / 2) x^{-1+\nu / 2} \exp \left(-x^{\nu / 2}\right)$, with $x$ related to $\varepsilon$ by $x$ $=\varepsilon\left(\widetilde{C}_{\nu} \Omega / \delta \omega_{0}\right)^{2 / \nu}$, for different number of holes $\nu=1,2,3$.

loss $\gamma_{0}$, the high frequency modes cannot be excited. It follows that the only relevant cavity modes are those with frequencies smaller than $\omega_{\max }$. Their number $M$ $\approx \omega_{\max }^{3} / 3 \omega_{0}^{2} \delta \omega_{0} \simeq(D / d)^{3}$ is $\gg 1$. From Eq. (11) the probability of no lasing $p_{1}(\varepsilon)$ can be cast in the form of Eq. (8) with the coefficient $\widetilde{C}_{1}=(8 / \pi)^{1 / 2} \ln \left(\omega_{\max } / \Omega\right)$ weakly dependent on the frequency cutoff $\omega_{\max }$. Figure 1 shows that the probability of lasing $1-p_{1}(\varepsilon)$ can be reasonably large even for extremely small values of the reduced pumping rate $\varepsilon$.

The quantity $1-p_{\nu}(\varepsilon)$ is the fraction of lasing cavities in an array at a given pumping rate $\varepsilon$. It is directly related to the probability distribution $T_{\nu}(\varepsilon)$ of the lasing threshold. Obviously $\int_{0}^{\varepsilon} d \varepsilon^{\prime} T_{\nu}\left(\varepsilon^{\prime}\right)=1-p_{\nu}(\varepsilon)$, hence $T_{\nu}(\varepsilon)=-d p_{\nu}(\varepsilon) /$ $\mathrm{d} \varepsilon$. We find from Eq. (8) that

$$
T_{\nu}(\varepsilon)=\frac{1}{2} \nu \widetilde{C}_{\nu}\left(\Omega / \delta \omega_{0}\right) \varepsilon^{-1+\nu / 2} \exp \left[-\widetilde{C}_{\nu}\left(\Omega / \delta \omega_{0}\right) \varepsilon^{\nu / 2}\right] .
$$

(Deviations which arise at $\varepsilon \gtrsim 1$ are unimportant.) The distribution is wide and in the single-hole case $\nu=1$ diverges as $\varepsilon \rightarrow+0$ (see right inset of Fig. 1). The average reduced threshold reads $\left\langle\varepsilon_{\nu}\right\rangle=\Gamma(1+2 / \nu)\left(\widetilde{C}_{\nu} \Omega / \delta \omega_{0}\right)^{-2 / \nu}$. It is smallest for $\nu=1$ and is indeed much smaller than 1 .

\section{EFFECTS OF NONZERO WALL RESISTIVITY}

A nonzero loss $\gamma_{*}$ from the resistivity of the cavity walls modifies the functions (10)-(12) by suppressing lasing for $\varepsilon<\gamma_{*} / \gamma_{0}$. The distribution of the lasing threshold remains wide, as long as $\gamma_{*} / \gamma_{0} \ll 1$, as we now show. Instead of Eq. (10) we have

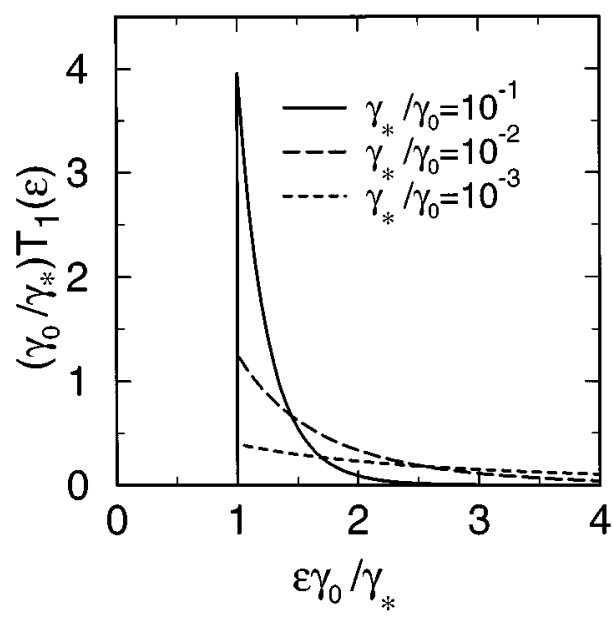

FIG. 2. Probability distribution of the lasing threshold in a cavity with small absorption in the boundary and a single hole $(\nu$ $=1$ ), computed as $-d p_{1}(\varepsilon) / d \varepsilon$ from Eq. (14). We chose $\Omega / \delta \omega_{0}$ $=10$ and took three values of $\gamma_{*} / \gamma_{0}$ such that $\gamma_{*} / \gamma_{0}$ is much smaller than, equal to, or much greater than $\left(\delta \omega_{0} / \Omega\right)^{2 / \nu}=10^{-2}$.

$$
p_{\nu}(\varepsilon)=\exp \left(\int_{\omega_{-}}^{\omega_{+}} d \omega \rho(\omega) \ln \int_{\left(g(\omega)-\gamma_{*}\right) / \bar{\gamma}(\omega)}^{\infty} d y P_{\nu}(y)\right),
$$

where $\omega_{-}<\omega_{+}$are the two positive frequencies such that $g\left(\omega_{ \pm}\right)=\gamma_{*}$. A nonzero value of $\gamma_{*}$ reduces the relevant frequency range to a narrow window around $\omega_{0}$. Therefore the modifications (1)-(3) of the preceding section become unnecessary even for the case $\nu=1$. Using the simple Lorentzian (4) for $g(\omega)$, instead of the more complicated expression (9), we find $\omega_{ \pm}=\omega_{0} \pm \Omega\left(\varepsilon \gamma_{0} / \gamma_{*}-1\right)^{1 / 2}$. Neglecting the $\omega$ dependence of $\rho(\omega), \bar{\gamma}(\omega)$ and using the small-argument behavior of the probability function $P_{\nu}(y)$, we reduce Eq. (13) to

$$
p_{\nu}(\varepsilon)=\exp \left[-C_{\nu}\left(\Omega / \delta \omega_{0}\right)\left(\gamma_{*} / \gamma_{0}\right)^{\nu / 2} f_{\nu}\left(\varepsilon \gamma_{0} / \gamma_{*}-1\right)\right]
$$

where $C_{\nu}$ is the numerical coefficient introduced in Eq. (7) and

$$
f_{\nu}(z)=\sqrt{z} \int_{-1}^{1} d y\left(\frac{1-y^{2}}{y^{2}+1 / z}\right)^{\nu / 2}
$$

can be expressed in terms of a hypergeometric function. In Fig. 2 we have plotted the distribution of the lasing threshold, $T_{\nu}(\varepsilon)=-d p_{\nu}(\varepsilon) / d \varepsilon$, for $\nu=1$ and different values of $\gamma_{*} / \gamma_{0}$. We will analyze two limiting regimes.

In the regime $\varepsilon \gamma_{0} / \gamma_{*} \gg 1$ and for $\nu>1$ we recover the expression (12) with the same constant $\widetilde{C}_{\nu}$. The value of $\widetilde{C}_{1}=C_{1} \ln \left(\varepsilon \gamma_{0} / \gamma_{*}\right)$ is different because of the different cutoff mechanism. Instead of having a weak logarithmic dependence on $\omega_{\max }$ it exhibits a weak logarithmic dependence on the pumping rate $\varepsilon$. This limiting case is statistically dominant if $\gamma_{*} / \gamma_{0} \ll\left(\delta \omega_{0} / \Omega\right)^{2 / \nu}$, because then the corrections to Eq. (12) at $\varepsilon \lesssim \gamma_{*} / \gamma_{0}$ have negligible statistical weight.

In the opposite regime, $\varepsilon \gamma_{0} / \gamma_{*}-1 \ll 1$, the threshold distribution differs significantly from Eq. (12), 


$$
\begin{aligned}
T_{\nu}(\varepsilon)= & \frac{1}{2}(1+\nu) A_{\nu}\left(\Omega / \delta \omega_{0}\right)\left(\gamma_{0} / \gamma_{*}\right)^{1 / 2} \\
& \times\left(\varepsilon-\gamma_{*} / \gamma_{0}\right)^{(\nu-1) / 2} \exp \left[-A_{\nu}\left(\Omega / \delta \omega_{0}\right)\left(\gamma_{0} / \gamma_{*}\right)^{1 / 2}\right. \\
& \left.\times\left(\varepsilon-\gamma_{*} / \gamma_{0}\right)^{(1+\nu) / 2}\right]
\end{aligned}
$$

[with a numerical coefficient $A_{\nu}=\sqrt{\pi} C_{\nu} \Gamma(1+\nu / 2) / \Gamma(3 / 2$ $+\nu / 2)]$. This regime is statistically dominant if $\gamma_{*} / \gamma_{0}$ $\gg\left(\delta \omega_{0} / \Omega\right)^{2 / \nu}$. The mean value of threshold is now close to $\gamma_{*} / \gamma_{0}$, but there are large fluctuations towards larger $\varepsilon$.

\section{AVERAGE NUMBER OF EXCITED MODES}

In this section we focus on the number of lasing modes beyond the lasing threshold for $\nu=1$ assuming $\gamma_{*}=0$. We assume that the parameters are such that many modes are above the threshold. This requires, in particular, $\varepsilon \gamma_{0} / \gamma_{*}$ $\gg 1$. In this case a nonzero value of $\gamma_{*}$ only leads to a redefinition of $\widetilde{C}_{1}$ because of the different cutoff mechanism. If the modes did not compete we could compute the average number of excited modes $\left\langle N_{\text {nc }}\right\rangle$ as

$$
\left\langle N_{\mathrm{nc}}\right\rangle=\int_{0}^{\omega_{\max }} d \omega \rho(\omega) \operatorname{erf} \sqrt{\frac{g(\omega)}{2 \bar{\gamma}(\omega)}} .
$$

For $\varepsilon<1$ it is given by $\left\langle N_{\mathrm{nc}}\right\rangle=\widetilde{C}_{1}\left(\Omega / \delta \omega_{0}\right) \varepsilon^{1 / 2}$. However, the modes do compete for a homogeneously broadened line because one of the modes can deplete the inversion, preventing another mode from being excited [18]. Multimode operation is still possible if different excited modes deplete the inversion in different spatial regions of the cavity [19,20]. We assume this mechanism of multimode generation, called spatial hole burning [21].

Let $n_{i}, \mathcal{N}(\vec{r})$ denote the number of photons in the mode $i$ and the density of population inversion between the lasing levels. Semiclassical rate equations read

$$
\begin{gathered}
\frac{d n_{i}}{d t}=-\gamma_{i} n_{i}+W_{i}\left(n_{i}+1\right) \int d \vec{r} \psi_{i}^{2}(\vec{r}) \mathcal{N}(\vec{r}), \\
\frac{d \mathcal{N}(\vec{r})}{d t}=\varepsilon R_{p_{0}} / V-w \mathcal{N}(\vec{r})-\mathcal{N}(\vec{r}) \sum_{i} W_{i} n_{i} \psi_{i}^{2}(\vec{r}) .
\end{gathered}
$$

Here $w$ is the nonradiative decay rate and $W_{i}$ is the rate of stimulated emission into mode $i$. The constant $W_{i}$ is related to the gain (9) in the corresponding mode, $W_{i}$ $=w g\left(\omega_{i}\right) / \varepsilon R_{p_{0}}$.

We restrict ourselves to a steady state solution. Let there be $N$ excited modes, $i_{1}, i_{2}, \ldots, i_{N}$. Because the number of photons in an excited mode is very large, we can approximate $n_{i_{k}}+1 \approx n_{i_{k}}$ in the right-hand side of Eq. (18). Eliminating the equilibrium population inversion density $\mathcal{N}(\vec{r})$, we get the following set of equations for the equilibrium mode populations $n_{i_{k}}$ :

$$
\left(-\gamma_{i_{k}}+\varepsilon R_{p_{0}} W_{i_{k}} \int \frac{d \vec{r}}{V} \frac{\psi_{i_{k}}^{2}(\vec{r})}{w+\sum_{j} W_{j} n_{j} \psi_{j}^{2}(\vec{r})}\right) n_{i_{k}}=0 .
$$

Nonexcited modes typically contain only few photons and can be omitted from the sum. We assume that we are not far beyond threshold, so that $w \gg \sum_{j} W_{j} n_{j} \psi_{j}^{2}(\vec{r})$, and we may expand the denominator in Eq. (20). We arrive at the following system of linear equations $(k=1, \ldots, N)$ :

$$
\frac{1}{\varepsilon R_{p_{0}}} \sum_{l=1}^{N} A_{i_{k} i_{l}} g\left(\omega_{i_{l}}\right) n_{i_{l}}=1-\frac{\gamma_{i_{k}}}{g\left(\omega_{i_{k}}\right)},
$$

subject to a constraint $n_{i_{k}}>0$.

So far we have followed the reasoning of Refs. [19,20]. Now we need to take into account randomness of coefficients in Eq. (21). Coefficients $A_{i_{k} i_{l}}$ are given by

$$
A_{i_{k} i_{l}}=\frac{1}{V} \int d \vec{r} \psi_{i_{k}}^{2}(\vec{r}) \psi_{i_{l}}^{2}(\vec{r})
$$

They are self-averaging quantities with negligibly small fluctuations around their mean $\left\langle A_{i_{k} i_{l}}\right\rangle=1+2 \delta_{i_{k} i_{l}}$, which follows from the independent Gaussian distributions for $\psi_{i}(\vec{r})$ [22]. Because the correlations between $A_{i_{k} i_{l}}$ 's and $\gamma_{i_{s}}$ 's are also negligibly small, we may substitute $A_{i_{k} i_{l}}=1+2 \delta_{i_{k} i_{l}}$ in Eq. (21). Without loss of generality we can assume that $\gamma_{i_{1}} / g\left(\omega_{i_{1}}\right) \leqslant \gamma_{i_{2}} / g\left(\omega_{i_{2}}\right) \leqslant \cdots \leqslant \gamma_{i_{N}} / g\left(\omega_{i_{N}}\right)$. Inverting the matrix $A_{i_{k} i_{l}}$ we find

$$
\frac{g\left(\omega_{i_{k}}\right) n_{i_{k}}}{\varepsilon R_{p_{0}}}=\frac{1}{N+2}-\frac{\gamma_{i_{k}}}{2 g\left(\omega_{i_{k}}\right)}+\frac{1}{2(N+2)} \sum_{l=1}^{N} \frac{\gamma_{i_{l}}}{g\left(\omega_{i_{l}}\right)} .
$$

The number of excited modes $N$ is restricted by the requirement that all $n_{i_{k}}$ 's should be positive. A necessary and sufficient condition is

$$
(2+N) \frac{\gamma_{i_{N}}}{g\left(\omega_{i_{N}}\right)}-\sum_{l=1}^{N} \frac{\gamma_{i_{l}}}{g\left(\omega_{i_{l}}\right)}<2 .
$$

Equation (24) can be used to determine the probability distribution of the number of excited modes, using the PorterThomas distribution (3) for the statistics of decay rates $\gamma_{i}$. In the region of parameters where $\langle N\rangle \gg 1$ this mean value can be found analytically from the continuous approximation of the condition (24),

$$
\left(2+\int_{0}^{\alpha_{\max }} d \alpha \sigma(\alpha)\right) \alpha_{\max }-\int_{0}^{\alpha_{\max }} d \alpha \alpha \sigma(\alpha)=2,
$$

with $\langle N\rangle=\int_{0}^{\alpha_{\max }} d \alpha \sigma(\alpha)$. The density $\sigma(\alpha)$ of the variables $\alpha_{i}=\gamma_{i} / g\left(\omega_{i}\right)$ is given by

$$
\begin{aligned}
\sigma(\alpha) & =\int_{0}^{\omega_{\max }} d \omega \rho(\omega) P_{1}[\alpha g(\omega) / \bar{\gamma}(\omega)] g(\omega) / \bar{\gamma}(\omega) \\
& =\frac{1}{2} \widetilde{C}_{1}\left(\Omega / \delta \omega_{0}\right) \alpha^{-1 / 2}
\end{aligned}
$$

It follows from Eq. (25) that $\langle N\rangle=\widetilde{C}_{1}\left(\Omega / \delta \omega_{0}\right) z$, where $z$ satisfies a cubic equation 


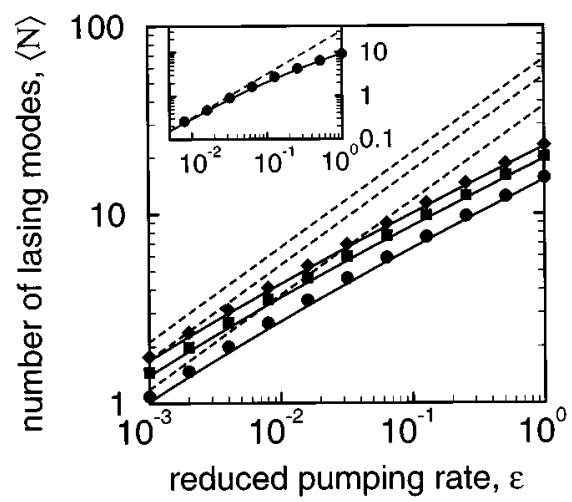

FIG. 3. Average number of excited modes $\langle N\rangle$ versus dimensionless pumping rate $\varepsilon$ (same parameters as in Fig. 1). The solid lines are the analytical result (27), the data points are a Monte Carlo average. The main plot corresponds to $\nu=1, M=10^{2}$ (circles), $M$ $=10^{3}$ (squares), $M=10^{4}$ (diamonds). Dashed lines represent the average number $\left\langle N_{\text {nc }}\right\rangle$ of noncompeting modes. The inset shows the case $\nu=2$ for $M \gg 1$. Note a drastic reduction in the number of excited modes.

$$
z^{2}+\frac{1}{3} \widetilde{C}_{1}\left(\Omega / \delta \omega_{0}\right) z^{3}=\varepsilon .
$$

To leading order in $1 /\langle N\rangle$ the term $z^{2}$ can be neglected, which yields a simple answer

$$
\langle N\rangle=3^{1 / 3}\left(\widetilde{C}_{1} \Omega / \delta \omega_{0}\right)^{2 / 3} \varepsilon^{1 / 3}=3^{1 / 3}\left\langle N_{\mathrm{nc}}\right\rangle^{2 / 3} .
$$

The general form of this result for any $\nu$ can be derived in a similar way, leading to $\left\langle N_{\mathrm{nc}}\right\rangle=\widetilde{C}_{\nu}\left(\Omega / \delta \omega_{0}\right) \varepsilon^{\nu / 2},\langle N\rangle=(\nu$ $+2)^{\nu /(\nu+2)}\left\langle N_{\mathrm{nc}}\right\rangle^{2 /(\nu+2)}$. These results are independent of $\gamma_{*}$ as long as $\gamma_{*} \ll \gamma_{0}$.
To test numerically the analytical results for $\langle N\rangle$, we did a Monte Carlo average over the Porter-Thomas distribution. For each of 2000 realizations, we ordered the modes in increasing order of the ratio loss over gain and found maximal $N$ satisfying Eq. (24). Results for $\langle N(\varepsilon)\rangle$ are in excellent agreement with the continuous approximation down to $\langle\mathrm{N}\rangle \sim 1$ (Fig. 3).

\section{CONCLUSION}

To summarize, we have considered lasing of a chaotic cavity coupled to the outside world via $\nu$ small holes. We assumed that the broadening of the cavity modes (due to leakage through the holes and absorption by the cavity walls) is less than their spacing and used a simple criterion "modal gain $\geqslant$ modal loss"' as the condition for a given mode to be excited. Natural unit of the pumping rate $R_{p_{0}}$ is defined such that "maximal gain = mean loss." Because of strong fluctuations of modal widths, the probability of lasing can be significantly large for much weaker pumping rates than $R_{p_{0}}$. The distribution of the lasing threshold turns out to be wide, with the mean much less than $R_{p_{0}}$. We have described the multimode operation as a result of spatial hole burning and found that the average number of excited modes is proportional to the power $\nu /(\nu+2)$ of the pumping rate.

\section{ACKNOWLEDGMENTS}

This work was supported by the Nederlandse Organisatie voor Wetenschappelijk Onderzoek (NWO) and the Stichting voor Fundamenteel Onderzoek der Materie (FOM).
[1] A. Lagendijk and B. A. van Tiggelen, Phys. Rep. 270, 143 (1996).

[2] N. M. Lawandy, R. M. Balachandran, A. S. L. Gomes, and E. Sauvain, Nature (London) 368, 436 (1994).

[3] W. L. Sha, C.-H. Liu, and R. R. Alfano, Opt. Lett. 19, 1922 (1994).

[4] D. S. Wiersma, M. P. van Albada, and A. Lagendijk, Phys. Rev. Lett. 75, 1739 (1995).

[5] P. Pradhan and N. Kumar, Phys. Rev. B 50, 9644 (1994).

[6] Z. Q. Zhang, Phys. Rev. B 52, 7960 (1995).

[7] C. W. J. Beenakker, J. C. J. Paasschens, and P. W. Brouwer, Phys. Rev. Lett. 76, 1368 (1996).

[8] J. C. J. Paasschens, T. Sh. Misirpashaev, and C. W. J. Beenakker, Phys. Rev. B 54, 11887 (1996).

[9] A. Yu. Zyuzin, Phys. Rev. E 51, 5274 (1995).

[10] A. Mekis, J. U. Nöckel, G. Chen, A. D. Stone, and R. K. Chang, Phys. Rev. Lett. 75, 2682 (1995).

[11] L. Bunimovich, G. Casati, and I. Guarneri, Phys. Rev. Lett. 77, 2941 (1996).

[12] H. Alt, H.-D. Gräf, H. L. Harney, R. Hofferbert, H. Lengeler, A. Richter, P. Schardt, and H. A. Weidenmüller, Phys. Rev. Lett. 74, 62 (1995).
[13] H. Alt, C. Dembowski, H.-D. Gräf, R. Hofferbert, H. Rehfeld, A. Richter, R. Schuhmann, and T. Weiland, Phys. Rev. Lett. 79, 1026 (1997).

[14] H. A. Bethe, Phys. Rev. 66, 163 (1944).

[15] M. V. Berry, J. Phys. A 10, 2083 (1977).

[16] A. Kudrolli, V. Kidambi, and S. Sridhar, Phys. Rev. Lett. 75, 822 (1995); V. N. Prigodin, N. Taniguchi, A. Kudrolli, V. Kidambi, and S. Sridhar, ibid. 75, 2392 (1995).

[17] T. A. Brody, J. Flores, J. B. French, P. A. Mello, A. Pandey, and S. S. M. Wong, Rev. Mod. Phys. 53, 385 (1981).

[18] A. Siegman, Lasers (University Science Books, Mill Valley, CA, 1986).

[19] H. Haken and H. Sauermann, Z. Phys. 173, 261 (1963).

[20] C. L. Tang, H. Statz, and G. A. deMars, J. Appl. Phys. 34, 2289 (1963).

[21] For a detailed treatment of spatial hole burning see H. Haken, Light (North-Holland, Amsterdam, 1985), Vol. 2, Chap. 4.

[22] Spatial correlations in $\psi_{i}(\vec{r})$ exist on the scale of the wavelength $\lambda$. These are irrelevant for the calculation of $A_{i j}$ when $D \gg \lambda$. 